\section{VARIACIÓN Y PREDICCIÓN DE LA DENSIDAD BÁSICA DE LA MADERA DE Cedrela odorata L.}

\section{VARIATION AND PREDICTION OF BASIC WOOD DENSITY IN Cedrela odorata L.}

\author{
Benito N. Gutiérrez-Vázquez ${ }^{1 \star}$, Eladio H. Cornejo- \\ Oviedo $^{2}$, Mario H. Gutiérrez-Vázquez ${ }^{3}$ y Martin Gómez- \\ Cárdenas $^{4}$
}

\begin{abstract}
${ }^{1}$ Centro Académico Regional Chiapas, Universidad Autónoma Agraria Antonio Narro, Cintalapa, Chiapas. ${ }^{2}$ Departamento Forestal, Universidad Autónoma Agraria Antonio Narro, Buenavista, Saltillo, Coahuila. ${ }^{3}$ Comisión Forestal Sustentable del Estado de Chiapas. ${ }^{4}$ Campo Experimental Valles Centrales de Oaxaca Instituto Nacional de Investigaciones Forestales Agrícolas y Pecuarias.
\end{abstract}

*Autor para correspondencia (bn_gutierrez@hotmail.com)

\section{RESUMEN}

En este estudio se determinó el patrón y la magnitud de la variación de la densidad básica de la madera de Cedrela odorata en cuatro localidades del Estado de Chiapas, México, y se desarrolló un modelo predictivo de la densidad total del árbol a partir de la densidad obtenida a la altura del diámetro normal. La densidad de la madera se determinó con el método empírico. La variación se cuantificó mediante análisis de varianza y de componentes de varianza. $C$. odorata presentó una densidad básica promedio de $0.34 \mathrm{gcm}^{-3}$, con diferencias $(P \leq 0.05)$ entre localidades y entre árboles dentro de las localidades. La localidad de Reforma presentó mayor densidad básica de la madera que la de Dr. Manuel Velasco Suárez II. También hubo diferencias $(P \leq 0.05)$ en la variación axial y radial. El modelo ajustado para estimar la densidad promedio del árbol fue: $d m=0.0930+0.7835 d n_{1.30}$, con una $\mathrm{R}^{2} \mathrm{de} 0.78$. Según el análisis de correlación, los árboles con mayor ICAD o diámetro tienden a tener densidades más altas.

Palabras clave: Cedrela odorata, densidad básica de la madera, patrones de variación, componentes de varianza.

\section{SUMMARY}

In this study we determined the pattern and the magnitude of wood density variation for Cedrela odorata in four locations in the State of Chiapas, México, and we developed a predictive model for whole tree density from density at one sample height. The density of the wood was determined with an empiric method. The variation was determined by an analysis of variance analysis and by the variance components. $C$. odorata showed a basic density average of $0.34 \mathrm{~g} \mathrm{~cm}^{-3}$, with differences $(P \leq 0.05)$ among localities and among trees inside the localities. In the locality of Reforma C. odorata presented higher wood density than in Dr. Manuel Velasco Suárez II. There was significant variation also $(\mathrm{P} \leq$ $0.05)$ across axial and radial positions in the trees. The adjusted model to estimate the density average of the tree was: $d m=0.0930+0.7835$ $d n_{1.30}$, with an $\mathrm{R}^{2}$ of 0.78 . According to the correlation analysis, the trees with bigger ICAD or diameter tend also to have a higher wood density.

Index words: Cedrela odorata, wood density, variation patterns, variance components.

\section{INTRODUCCIÓN}

En México, durante muchos años sólo dos especies de madera tropical se comercializaron, Cedrela odorata L. y Swietenia macrophylla King (Bárcenas, 1995). Sin embargo, el conocimiento de las propiedades de la madera se basa generalmente en conocimiento empírico, lo que incita a un uso inapropiado. La densidad de la madera es una propiedad universalmente utilizada como índice de calidad (Barnett y Jeronimidis, 2003). Esta propiedad puede variar ampliamente dentro de un árbol, desde la médula hacia el exterior o axialmente desde la base del tronco hacia el ápice (Zobel y Talbert 1988). Conocer estas variaciones permitiría realizar muestreos de madera representativos del valor del árbol completo y reconocer diferencias de calidad en relación con los usos (Downes et al., 1997).

Los modelos de variación radial y axial de la densidad ya han sido estudiados en especies latifoliadas como Populus spp., Eucalyptus spp., Acacia spp. y Salix spp. Los resultados indican que la variación axial de la densidad es de menor magnitud que la variación radial (Wilken, 1988). Los estudios manifiestan un aumento de la densidad desde la base al ápice (Igartúa et al., 2003). Para estimar la densidad del árbol completo a partir de un único punto de muestreo, debe hacerse una regresión entre todos los valores de densidad del árbol y la correspondiente a dicha altura (Zobel y Talbert, 1988). Existen trabajos de este tipo para especies comerciales como Eucalyptus globulus Labill. (Igartúa et al., 2003), E. grandis Hill (ex Maiden) (Clark, 2001), E. nitens Deane et Maiden (Raymond y Muneri, 2001), Salix babylonica var. sacramenta $\mathrm{H}$. (Villegas y Marlats, 2005), y para un clon de Populus deltoides (Diaz et al., 2010).

Los objetivos de este estudio fueron determinar el patrón y la magnitud de la variación de la densidad básica de la madera de Cedrela odorata y desarrollar un modelo predictivo de la densidad total del árbol a partir de la densidad obtenida a la altura del diámetro normal.

\section{MATERIALES Y MÉTODOS}

El estudio se llevó a cabo en cuatro localidades del Estado de Chiapas, México donde hay Cedrela odorata, ubicadas en Paso Hondo, Municipio de Frontera Comalapa; Reforma, Municipio de Bejucal de Ocampo; Dr. Manuel Velasco Suárez II, Municipio Ocozocoautla; y Ribera de Chalchi, Municipio de Venustiano Carranza (Cuadro 1).

En cada localidad se eligieron sitios para la colecta de muestras de madera de los árboles, y en cada sitio se eligieron de siete a diez árboles. En todos los casos se cuidó que la distancia entre árboles fuera mayor a $50 \mathrm{~m}$. A cada árbol se le midió diámetro a la base del fuste, diámetro normal (a 
Cuadro 1. Descripción climática y topográfica de las localidades con Cedrela odorata en el Estado de Chiapas, México.

\begin{tabular}{lccccccc}
\hline Localidad & $\begin{array}{c}\text { Latitud } \\
\text { Norte }\end{array}$ & $\begin{array}{c}\text { Longitud } \\
\text { Oeste }\end{array}$ & $\begin{array}{c}\text { Altitud } \\
(\mathrm{m})\end{array}$ & $\begin{array}{c}\text { Precip. media } \\
\text { anual }(\mathrm{mm})\end{array}$ & $\begin{array}{c}\text { Temp. media } \\
\text { anual }\left({ }^{\circ} \mathrm{C}\right)\end{array}$ & $\begin{array}{c}\text { Tipo de } \\
\text { suelo }\end{array}$ & $\begin{array}{c}\text { Edad promedio } \\
\text { (años) }\end{array}$ \\
\hline Paso Hondo & $15^{\circ} 41^{\prime} 17^{\prime \prime}$ & $92^{\circ} 03^{\prime} 22^{\prime \prime}$ & 801 & 1000 & 23 & Rendzina & 20 \\
Reforma & $15^{\circ} 32^{\prime} 05^{\prime \prime}$ & $92^{\circ} 07^{\prime} 16^{\prime \prime}$ & 801 & 1350 & 23 & $\begin{array}{c}\text { Fluvisol } \\
\text { eutrico }\end{array}$ & 24 \\
$\begin{array}{l}\text { Dr. M. Velas- } \\
\text { co Suárez }\end{array}$ & $16^{\circ} 57^{\prime} 20^{\prime \prime}$ & $93^{\circ} 23^{\prime} 17^{\prime \prime}$ & 401 & 1600 & 25 & $\begin{array}{c}\text { Acrisol } \\
\text { humico }\end{array}$ & 16 \\
$\begin{array}{l}\text { Ribera de } \\
\text { Chalchi }\end{array}$ & $16^{\circ} 19^{\prime} 52^{\prime \prime}$ & $92^{\circ} 38^{\prime} 26^{\prime \prime}$ & 601 & 1350 & 25 & $\begin{array}{c}\text { Feozem } \\
\text { haplico }\end{array}$ & 26 \\
\hline
\end{tabular}

$1.30 \mathrm{~m}$ de altura), altura total y altura de fuste limpio. De cada árbol seleccionado se extrajeron muestras individuales a cuatro alturas diferentes $(0.30 \mathrm{~m}, 1.30 \mathrm{~m}$, a $40 \%$ y a 60 $\%$ de la altura total del árbol). La muestra consistió en un cilindro de madera extraída con un taladro de Pressler ${ }^{\circledR}$.

Cada muestra se identificó con número de localidad, número de árbol y altura de extracción, y se guardó en un cilindro de plástico para su protección durante el traslado al laboratorio. Se determinó la densidad básica (peso anhidro/volumen verde) de la madera, con el método empírico propuesto por Valencia y Vargas (1997). A diferencia del proceso de determinación de la variación axial donde se utilizó la muestra completa, para la variación radial fue necesario seccionar cada muestra o porción de madera en dos submuestras de $5 \mathrm{~cm}$, una de la sección cercana a la médula y otra de la sección cercan a la corteza. Además se calculó el incremento corriente anual en diámetro (ICAD). Se hizo un análisis de correlación de Pearson entre las variables: densidad media del árbol ( $\mathrm{dm}$ ), altura total, altura de fuste limpio, diámetro basal, diámetro normal, edad e ICAD.

Para estudiar los efectos de localidad, de árboles dentro de localidad y de muestras dentro de cada árbol, se consideró un diseño completamente al azar con efecto anidado o jerárquico, que corresponde al siguiente modelo estadístico:

$$
Y i j=\mu+L i+A i(j)+M i(j k)+\varepsilon i(j k l)
$$

donde: $Y i j=$ valor de la observación; $\mu=$ media general; $L i$ $=$ efecto de la $i$-ésima localidad; $A i(j)=$ efecto del $j$-ésimo árbol dentro de la $i$-ésima localidad; $M i(j k)=$ efecto de la $k$-ésima muestra dentro del $j$-ésimo árbol de la $i$-ésima localidad; $\varepsilon i(j k l)=$ error experimental (submuestras): $i=1$, 2, 3, 4 (número de localidades); $j=1,2,3,4 \ldots$ (número de árboles en cada localidad); $k=1,2,3,4$ (número de muestras en cada árbol); $l=1,2$ (número de submuestras en cada muestra).
De acuerdo con este modelo se hizo un análisis de varianza con el PROC GLM y de componentes de varianza con el PROC VARCOMP para la variable densidad de la madera, con el paquete estadístico SAS versión 9.1 (SAS Institute, 1999). Además se hizo ajuste del modelo de predicción con el PROC REG y un análisis de correlación con el PROC CORR.

\section{RESULTADOS Y DISCUSIÓN}

El valor promedio de la densidad básica de la madera de todos los individuos (peso anhidro/volumen verde) fue de $0.34 \mathrm{~g} \mathrm{~cm}^{-3}$, con valores mínimos y máximos de 0.27 y 0.44 $\mathrm{g} \mathrm{cm}^{-3} \mathrm{y}$ un coeficiente de variación de $11.66 \%$. El valor promedio estimado de la densidad de la madera es ligeramente menor a las densidades reportadas para $C$. odorata de la Selva Lacandona del Estado de Chiapas, de $0.36 \mathrm{~g} \mathrm{~cm}^{-3}$ por Bárcenas (1995), y de $0.37 \mathrm{~g} \mathrm{~cm}^{-3}$ por Bárcenas y Dávalos (1999). El valor aquí estimado de la densidad permitiría clasificarla como madera "liviana", de acuerdo con la clasificación de Bárcenas et al. (1995), quien propusieron utilizarla para usos no estructurales en exteriores, ebanistería y productos torneados.

El análisis de varianza mostró diferencias significativas $(\mathrm{P}<0.01)$ entre localidades, entre árboles dentro de localidades y entre muestras dentro de los árboles de las localidades, para la densidad de la madera. Estos resultados muestran que existe efecto de la localidad y dentro de ella. A su vez, el análisis de componentes de varianza muestra que de la variación total de la densidad de la madera, $0.32 \%$ es atribuible al efecto de diferencias entre localidades, $18.99 \%$ al efecto de diferencias entre árboles dentro de localidades, $16.96 \%$ al efecto de diferencias entre las muestras dentro de los árboles de las localidades, y $63.70 \%$ al error o a una fuente no identificada (Cuadro 2).

Lo anterior es de interés si se considera que se trata de una característica de valor económico y de alta heredabilidad, 
Cuadro 2. Cuadrados medios y componentes de varianza de la densidad de la madera de Cedrela odorata crecida en el estado de Chiapas, en función de la localidad, del árbol y de la posición en el árbol.

\begin{tabular}{lrcccc}
\hline FV & gl & CM & Fc & $\operatorname{Pr}>$ F & Componentes de varianza (\%) \\
\hline Localidad & 3 & 0.0102 & 4.26 & $0.0067^{\star *}$ & 0.32 \\
Arb (Loc) & 28 & 0.0099 & 4.15 & $0.0001^{\star *}$ & 18.99 \\
Mue (Arb/Loc) & 96 & 0.0040 & 1.68 & $0.0032^{\star *}$ & 16.96 \\
Error & 127 & 0.0023 & & & 63.70
\end{tabular}

$\mathrm{FV}=$ fuente de variación; $\mathrm{gl}=$ grados de libertad; $\mathrm{CM}=$ cuadrados medios; $\mathrm{Fc}=$ valor calculado de $\mathrm{F} ; \mathrm{Pr}>\mathrm{F}=$ probabilidad de error tipo I $(\alpha)$; ** Significativo al nivel de probabilidad de $\mathrm{P}<0.01$; Arb (Loc) = árbol dentro de la localidad; Mue (Arb/Loc) = muestras dentro del árbol de la localidad.

$\begin{aligned} & \text { Cuadro 3. Prueba de medias para la densidad básica de la madera de Cedrela odorata en cuat } \\
& \text { localidades del Estado de Chiapas, México. }\end{aligned}$
\begin{tabular}{lll}
\hline Localidad & $\mathrm{N}$ & Densidad básica media $\left(\mathrm{g} \mathrm{cm}^{-3}\right)$ \\
\hline Reforma & 56 & $0.3544 \mathrm{a}$ \\
Ribera de Chalchi & 64 & $0.3446 \mathrm{ab}$ \\
Paso Hondo & 79 & $0.3415 \mathrm{ab}$ \\
Dr. M. Velasco Suárez II & 56 & $0.3222 \mathrm{~b}$ \\
\hline
\end{tabular}

que podría ser utilizada en programas de cruzamiento y selección. Dado que la variación atribuible a localidades es mínima, es posible que la variación asociada con la adaptabilidad de la especie podría ser no significativa, y entonces la selección puede ser dirigida a nivel de árbol, donde se registraron los valores más altos de componentes de varianza y por ende la variabilidad está más asociada con la heredabilidad a nivel individual (Zobel y Talbert, 1988).

En este caso la localidad de Reforma, Municipio de Bejucal de Ocampo presentó mayor densidad básica de la madera, que la población de Dr. Manuel Velasco Suárez II, Municipio de Ocozocoautla (Cuadro 3), aun que ello no significa que este efecto sea heredable.

Con respecto a la variación axial se encontraron diferencias significativas $(\mathrm{P}<0.01)$ entre las alturas a las cuales se tomaron las muestras. La altura de $60 \%$ fue la que mayor densidad $\left(0.375 \mathrm{~g} \mathrm{~cm}^{-3}\right)$ que las alturas inferiores $40 \%$, a $1.30 \mathrm{~m}$ y a $0.30 \mathrm{~m}$ cuyas densidades fueron $0.345,0.335$ y $0.316 \mathrm{~g} \mathrm{~cm}^{-3}$, respectivamente; estas últimas tres sin diferencias significativas entre ellas. El patrón de variación axial hallado mostró un aumento de los valores de densidad desde la base del árbol hacia el ápice. Este patrón coincide con el encontrado por Díaz et al. (2010) en un clon de Populus deltoides, y con el reportado por Gutiérrez y Baonza (2001) para 25 clones de álamo.
En relación con la variación radial se encontraron diferencias significativas $(P<0.01)$ entre las dos secciones evaluadas. La sección cercana a la corteza presentó mayor densidad básica de la madera $\left(0.383 \mathrm{~g} \mathrm{~cm}^{-3}\right)$ que la sección cercana a la médula $\left(0.323 \mathrm{~g} \mathrm{~cm}^{-3}\right)$. Esta variación radial de la densidad básica en la madera de C. odorata es similar a las reportadas para especies latifoliadas, como el estudio de Arroyo (1983) en latifoliadas y el de Oliveira et al. (2005) en cinco especies de eucalipto de Brasil. El incremento de la densidad de la madera con la distancia radial puede explicarse desde el punto de vista fisiológico por el crecimiento del árbol, que en los primeros años utiliza los materiales fotosintetizados para crear nuevas células y deja pocos sustratos para el engrosamiento de la pared celular. En cambio, en la etapa de madurez existe mayor cantidad de materia fotosintetizada destinada a la síntesis de la pared celular, lo que se traduce en un incremento de la densidad de la madera (Moglia y López, 2001).

El modelo ajustado para estimar la densidad promedio del árbol ( $\mathrm{dm}$ ) con base en la densidad determinada a la altura del diámetro normal a $1.30 \mathrm{~m}\left(\right.$ den $\left._{1.30}\right)$ fue: $d m=0.0930+0.7835 d e n_{1.30}$, ecuación lineal simple que se eligió por ser la de mayor $\mathrm{R}^{2}$ de 0.78 . Esta ecuación permite una rápida determinación de la densidad a una altura de fácil acceso y que se puede hacer por medios no destructivos. El modelo aquí desarrollado presenta mejor ajuste que el generado por Busnardo (Com. 
personal $)^{1}$ para Eucalyptus grandis con una $\mathrm{R}^{2}$ de 0.53 , y menor $\mathrm{R}^{2}$ que los modelos generados por Villegas y Marlats (2005) para Salix sp. con $\mathrm{R}^{2}$ de 0.80 , por Igartúa et al. (2003) para E. globulus con $\mathrm{R}^{2}$ de 0.80 , por Bhat et al. (1990) para E. grandis con $\mathrm{R}^{2}$ de 0.90 , por Beaudoin et al. (1992) para Populus sp. con $\mathrm{R}^{2}$ de 0.93 , y por Díaz et al. (2010) para un clon de Populus deltoides con $\mathrm{R}^{2}$ de 0.92 , si bien todos los modelos fueron significativos $(\mathrm{P}<0.05)$.

El análisis de correlación arrojó que la densidad promedio se relaciona de manera positiva con el ICAD $(r=0.511)$, el diámetro basal $(\mathrm{r}=0.507)$ y con el diámetro normal $(\mathrm{r}=$ $0.473)$, todas de modo significativo $(\mathrm{P}<0.05)$. Es decir, los árboles que presentan mayor ICAD o diámetro son los que tienden a mostrar las densidades más altas. Al igual que en este estudio, Díaz et al. (2010) encontraron en Populus deltoides que la densidad tomada a $1 \mathrm{~m}$ en el fuste se relacionó con la densidad media del árbol con $\mathrm{r}=0.87(\mathrm{P}<0.05)$; Beaudoin et al. (1992) también encontraron correlación significativa $(r=0.96 ; \mathrm{P}<0.05)$ en clones de $P$. canadensis.

\section{CONCLUSIÓN}

La relación positiva encontrada entre la densidad media del árbol y el ICAD, el diámetro basal y normal, sugiere la posibilidad seleccionar y producir genotipos de Cedrela odorata de mayor densidad en la madera producida.

\section{BIBLIOGRAFÍA}

Arroyo J (1983) Propiedades Físico-mecánicas de la Madera. Facultad de Ciencias Forestales y Ambientales. Universidad de Los Andes. Mérida, Venezuela. 197 p.

Bhat K M, K V Bhat, T K Dhamoradan (1990) Wood density and fibre

${ }^{1}$ Busnardo C A, J V Gonzaga, C E B Foelkel, S Menochelli (1987) Em busca da qualidade ideal da madeira do eucalipto para produção de celulosa IV. Altura ideal de amostragem para avaliação da densidade basica media para arvore de Eucalyptus grandis. XX Congreso Anual da ABCPSemana do Papel, San Pablo, Brasil. pp:17-33. length of Eucalyptus grandis grown in Kerala, India. Wood Fiber Sci. 22:54-61.

Bárcenas P G M (1995) Caracterización tecnológica de veinte especies maderables de la Selva Lacandona, Chis., México. Madera y Bosques 1:9-38.

Bárcenas P G M, R Dávalos S (1999) Importancia de la lignina en las contracciones de la madera: Revisión bibliográfica. Madera y Bosques 5:13-26.

Barnett J R, G Jeronimidis (2003) Wood Quality and its Biological Basis. CRC Press. Boca Ratón, USA. 226 p.

Beaudoin M, R E Hernandez, A Koubaa, J Poliquin (1992) Interclonal, intraclonal and within tree variation in wood density of poplar hybrid clones. Wood Fiber Sci. 24:147-153.

Clark N (2001) Longitudinal density variation in irrigated hardwoods. Appita J. 54:49-53.

Díaz G, S Monteoliva, J Álvarez, E Fernández T (2010) Populus deltoides 'Australiano 129/60': variación axial de la densidad y desarrollo de un modelo predictivo de la densidad del árbol completo. Bosque 31:65-72.

Downes G M, I Hudson, C Raymond, A Dean, A Micheli, L Schimlek, R Evans, A Muneri (1997) Sampling Eucalyptus for wood and fibre properties. Australia. CSIRO Publishing. $132 \mathrm{p}$.

Igartúa D V, S Monteoliva, M G Monterrubianesi, M S Villegas (2003) Basic density and fibre length at breast height of Eucalyptus globulus ssp. globulus for parameter prediction of the whole tree. IAWA J. 24:173-184.

Moglia J, C López (2001) Tendencias de variación radial del leño de Aspidosperma quebracho blanco. Inv. Agr. Sist. Rec. For. 10:69-79.

Oliveira J, J Hellmeister, M Filho (2005) Variação do teor de umidade e da densidade básica na madeira de sete espécies de Eucalipto. Àrvore 29:115-127.

Raymond C A, A Muneri (2001) Nondestructive sampling of Eucalyptus globulus and E. nitens for wood properties. Wood Sci. Tech. 35:27-39.

SAS Institute (1999) SAS/STAT Guide for Personal Computers. Version 8. Cary, NC. USA. 518 p.

Valencia M S, J J Vargas H (1997) Método empírico para estimar la densidad básica en muestras pequeñas de madera. Madera y Bosques 3:81-87.

Villegas M S, R M Marlats (2005) Altura de extracción de la muestra para evaluación de densidad básica y blancura en madera de Salix $s p$. Bosque 26:121-132.

Wilken J (1988) Variation in wood anatomy within species of Eucaliptus. IAWA Bull. 9:13-23.

Zobel B, J Talbert (1988) Técnicas de mejoramiento genético de árboles forestales. Ed. Limusa. Buenos Aires, Argentina. 545 p. 